\title{
Brigatinib versus other second-generation ALK inhibitors as initial treatment of anaplastic lymphoma kinase positive non-small cell lung cancer with deep phenotyping: study protocol of the ABP trial
}

Petros Christopoulos ${ }^{1,2}$, Farastuk Bozorgmehr ${ }^{1,2}$, Lena Brückner ${ }^{1,2}$, Inn Chung ${ }^{1,2}$, Johannes Krisam ${ }^{3}$, Marc A. Schneider ${ }^{2,4}$, Albrecht Stenzinger ${ }^{2,5}$, Regina Eickhoff ${ }^{6}$, Daniel W. Mueller ${ }^{6}$ and Michael Thomas ${ }^{1,2^{*}}$ (D)

\begin{abstract}
Background: Availability of potent anaplastic lymphoma kinase (ALK) tyrosine kinase inhibitors (TKI) has pushed the median survival of ALK ${ }^{+}$non-smallcell lung cancer (NSCLC) patients to over five years. In particular, secondgeneration ALK TKI have demonstrated superiority compared to the first-generation compound crizotinib and are meanwhile standard first-line treatment. However, clinical courses of individual patients vary widely, with secondary development of drug resistance and intracranial progression remaining important problems. While these limitations highlight the need for better disease monitoring and additional therapeutic tools, molecular tumor features are increasingly recognized as crucial determinants of clinical outcome. This trial aims to optimize management of $\mathrm{ALK}^{+}$NSCLC by analyzing the efficacy of second-generation ALK inhibitors in conjunction with deep longitudinal phenotyping across two treatment lines.
\end{abstract}

\footnotetext{
*Correspondence: michael.thomas@med.uni-heidelberg.de

'Department of Thoracic Oncology, Thoraxklinik at University Hospital of Heidelberg, Röntgenstraße 1, 69126 Heidelberg, Germany

${ }^{2}$ Translational Lung Research Center Heidelberg TLRCH, Member of the German Center for Lung Research DZL, Im Neuenheimer Feld 156, 69120 Heidelberg, Germany

Full list of author information is available at the end of the article
}

(c) The Author(s). 2021 Open Access This article is licensed under a Creative Commons Attribution 4.0 International License, which permits use, sharing, adaptation, distribution and reproduction in any medium or format, as long as you give appropriate credit to the original author(s) and the source, provide a link to the Creative Commons licence, and indicate if changes were made. The images or other third party material in this article are included in the article's Creative Commons licence, unless indicated otherwise in a credit line to the material. If material is not included in the article's Creative Commons licence and your intended use is not permitted by statutory regulation or exceeds the permitted use, you will need to obtain permission directly from the copyright holder. To view a copy of this licence, visit http://creativecommons.org/licenses/by/4.0/ The Creative Commons Public Domain Dedication waiver (http://creativecommons.org/publicdomain/zero/1.0/) applies to the data made available in this article, unless otherwise stated in a credit line to the data. 
Methods/design: In this exploratory prospective phase II clinical trial, newly diagnosed ALK+ NSCLC patients will be randomized into two treatment arms, stratified by presence of brain metastases and ECOG performance status: brigatinib (experimental arm) vs. any other approved second-generation ALK TKI. Tumor tissue and blood samples will be collected for biomarker analysis at the beginning and throughout the study period to investigate baseline molecular tumor properties and analyze the development of acquired drug resistance. In addition, participating investigators and patients will have the possibility of fast-track molecular tumor and ctDNA profiling at the time of disease progression using state-of-the-art next-generation sequencing (NGS), in order to support decisions regarding next-line therapy.

Discussion: Besides supporting therapeutic decisions for enrolled patients, the ABP trial primarily aims to deepen the understanding of the underlying biology and facilitate development of a framework for individualized management of ALK NSCLC according to molecular features. Patients with low molecular risk and the perspective of a "chronic disease" will be distinguished from "high-risk" cases, molecular properties of which will be utilized to elaborate improved methods of non-invasive monitoring and novel preclinical models in order to advance therapeutic strategies.

Trial registration: Clinicaltrials.gov, NCT04318938. Registered March 182,020, https://www.clinicaltrials.gov/ct2/show/ NCT04318938

Eudra-CT, 2019-001828-36. Registered September 302,019, https://www.clinicaltrialsregister.eu/ctr-search/search?query= 2019-001828-36

Keywords: Non-small cell lung cancer, Anaplastic lymphoma kinase, ALK ${ }^{+}$NSCLC, Tyrosine kinase inhibitors (TKI), Brigatinib, Resistance mutations, Molecular risk

\section{Background}

Non-small cell lung cancer (NSCLC) is the leading cause of cancer-related mortality worldwide with most patients diagnosed at an advanced, incurable stage [1-3]. Over the past years, advanced molecular diagnostics have revealed distinct driver molecular alterations in about 15$20 \%$ of these tumors, which also constitute particular therapeutic susceptibilities. Targeted treatment with tyrosine kinase inhibitors (TKI) has consistently demonstrated superior efficacy and tolerability compared to conventional chemotherapy in eligible patients and is meanwhile the standard first-line option.

$A L K$ (anaplastic lymphoma kinase)-positive tumors comprise approximately $5 \%$ of metastatic NSCLC, mainly adenocarcinomas of younger, non-smoker patients $[4,5]$, and are unique in many aspects: They typically have a lower number of genetic alterations compared to other NSCLC subtypes, as partly reflected by a mean tumor mutational burden (TMB) uniquely below 3 mut/Mb $[6,7]$. $\mathrm{ALK}^{+}$NSCLC patients require the most sophisticated management at present, i.e., high-level expertise and close cooperation between medical oncology, interventional pneumology, radiology, thoracic surgery, radiation oncology, as well as molecular pathology over several years, and enjoy the best outcome. Even the first-generation compound crizotinib results in longer overall survival (OS) for $\mathrm{ALK}^{+}$NSCLC patients compared to their TKI-treated $\mathrm{EGFR}^{+}$counterparts (for example $>46$ months in the PROFILE 1014 study vs. 39 and 32 months in the two arms of the
FLAURA trial), while sequential ALK TKI, particularly with upfront administration of second-generation inhibitors such as alectinib, confer a median OS over five years, which is certainly one of the greatest successes in modern thoracic oncology [6, 8-10]. First-line administration of the newer second-generation inhibitor brigatinib has produced comparable results to alectinib in the randomized phase III ALTA-1 L trial [11, 12], and both substances are currently initial treatments of choice [13, 14]. An important advantage of both compounds is enhanced intracranial efficacy, with brain overall response rates (iORR) of approximately $80 \%$ and rates of intracranial progression $<10 \%$ per year, compared to $30-50 \%$ and approximately $20 \%$ with the first-generation inhibitor crizotinib, respectively. Very recently, interim results from the CROWN trial, comparing lorlatinib against crizotinib in the first line, have been reported [15]. With a median follow-up of 18 months, data are still immature, but the hazard ratio (HR) of 0.28 for progression-free survival (PFS) in favor of the third-generation TKI lorlatinib indicates that this compound will probably also play a role as upfront therapy in the future. At the same time, the generally favorable outcome of $\mathrm{ALK}^{+}$NSCLC facilitates identification of special patient subsets with earlier treatment failure and higher risk of death. Currently, high-risk $\mathrm{ALK}^{+}$disease comprises three main groups: Tumors with the EML4-ALK fusion variant 3 (V3), whose shorter structure lowers TKI sensitivity and increases metastatic potential [16-20], tumors with TP53 mutations, either at initial diagnosis $[21,22]$ or at 
the time of TKI failure [23], and "double-positive patients", who have the worst outcome [22], as also recently demonstrated in the context of the randomized phase 3 ALTA-1 L trial [24]. Importantly, repeat molecular profiling based on tissue or liquid rebiopsies at the time of disease progression can identify actionable resistance mutations and guide individualized selection of next-line targeted therapies with higher clinical benefit for the patient $[25,26,27]$.

The aim of this trial is to optimize management of $\mathrm{ALK}^{+}$NSCLC by analyzing the efficacy of brigatinib and other ALK TKI in conjunction with deep clinical and molecular patient phenotyping across two treatment lines.

\section{Methods/design}

\section{Study design}

This is a prospective, randomized, open-label, multicenter phase II trial comparing brigatinib vs. other secondgeneration ALK inhibitors as first-line therapy for $\mathrm{ALK}^{+}$ NSCLC in conjunction with deep patient profiling across two treatment lines.

\section{Study setting}

The ABP trial will recruit patients from currently 24 participating centers across Germany over a period of 36 months. Recruitment started in June 2020. A full list of sites is available at clinicaltrials.gov (NCT04318938).

\section{Study objectives and endpoints}

The primary objective of this trial is a descriptive comparison of the efficacy of brigatinib vs. other approved second-generation ALK TKI in first-line treatment of locally advanced or metastatic $\mathrm{ALK}^{+}$NSCLC, as assessed by progression-free survival (PFS1) according to RECIST 1.1 [28]. Secondary objectives are treatment efficacy in the first and second line, assessed by the PFS of secondline therapy (PFS2), time-to-next treatment in the first line (TNT1), second line (TNT2) and until start of the third line (TNT1/2), as well as by the overall survival (OS). Moreover, CNS efficacy of first- and second-line treatment will be evaluated according to RECIST 1.1 regarding iORR, intracranial duration of response (iDOR) and time-to-intracranial progression (iTTP).

Safety in both treatment arms will be evaluated with regard to incidence and severity of adverse events (AE), while quality of life (QoL) will be assessed using the validated questionnaires SF-12 and EORTC-QLQ-BN20 (the latter in case of brain metastases, only).

Exploratory objectives are typing of $A L K$ fusion variants, assessment of TP53 status, profiling of resistance mechanisms at the failure of first-line treatment, and their relationship with efficacy endpoints. Furthermore, the potential clinical utility of cerebrospinal fluid ctDNA analysis will be assessed for cases with isolated intracranial progression.

\section{Characteristics of participants}

A total of 116 adult patients with newly diagnosed $\mathrm{ALK}^{+}$ NSCLC, either locally advanced (stage III) and not suitable for curative treatment, or metastatic (stage IV), will be enrolled into this study after written informed consent. The full list of inclusion and exclusion criteria is given in Table 1. ALK rearrangement will be determined locally by one or more ALK assays that are approved in Germany and/or have been validated within the German Network for Genomic Medicine, e.g. RNA-based NGS, fluorescence in situ hybridization using the ZytoLight SPEC ALK probe (ZytoVision GmbH, Bremerhaven, Germany) and immunohistochemistry using the D5F3 clone (Roche, Mannheim, Germany) [18, 29-31].

In addition, participants must have at least one measurable site of disease as defined by RECIST 1.1, sufficient ECOG performance status $(\leq 2)$, as well as adequate bone marrow, hepatic and renal function. Participants may not have received prior systemic therapy for $\mathrm{ALK}^{+}$ NSCLC, however up to two cycles of chemotherapy as well as stereotactic radiotherapy are allowed to accommodate for the fact that in some cases treatment might be initiated in an urgent manner before availability of molecular results. Further exclusion criteria include pulmonary interstitial disease, pneumonitis, uncontrolled hypertension and cardiovascular disease, significant infections or any other medical condition that may potentially compromise patient safety or hamper study completion.

\section{Study procedures}

Patients will be randomized into the experimental (brigatinib) or standard arm in a 1:1 ratio. The variance minimization method will be used for patient allocation, adjusting for the confounders brain metastases (presence vs. absence of brain metastases at baseline) and ECOG performance status $(0-1$ vs. 2$)$ in order to ensure better comparability between intervention groups. Overall, 58 patients will be enrolled per treatment arm.

\section{Treatment}

Patients in standard arm A are treated in the first line with any approved second-generation TKI other than brigatinib (currently alectinib or ceritinib) according to the investigator's choice, followed by second-line treatment with another approved ALK TKI of the investigator's choice. Patients in the experimental arm $\mathrm{B}$ receive brigatinib in the first line, followed by any other approved ALK TKI in the second line according to the investigator's choice (Fig. 1). It is recommended that the choice of second-line TKI takes into account the 
Table 1 Inclusion and exclusion criteria

\section{INCLUSION CRIT • Fully informed written consent and any locally-required authorization (EU Data Privacy Directive) given by the patient}

- Male or female $\geq 18$ years of age

- Histologically confirmed locally advanced (stage III) and not suitable for curative treatment, i.e. R0 operation or definitive chemo-/radiation, or metastatic (stage IV) ALK NSCLC

NOTE: Documentation of ALK rearrangement confirmed at baseline by a positive result of any ALK assay approved in Germany [i.e. positivity for at least one of the three: immunohistochemistry (IHC), NGS, fluorescence in situ hybridization (FISH)]. Treatment may be started based on a local $\mathrm{ALK}^{+}$test result, but subsequent central molecular profiling of the baseline biopsy, incl. determination of ALK variant and TP53 status, should be made possible for all patients.

- No prior therapy for metastatic ALK ${ }^{+}$NSCLC including ALK inhibitors (up to two cycles of chemotherapy as well as cerebra irradiation before inclusion in the study are acceptable)

- At least 1 measurable (i.e., target) lesion according to RECIST 1.1

- ECOG performance status $\leq 2$

- Adequate organ function, as determined by:

- Total bilirubin $\leq 1.5 x$ the upper limit of the normal range (ULN) $(<3 x$ the ULN in case of Gilbert's disease)

- Estimated glomerular filtration rate $\geq 30 \mathrm{~mL} /$ minute $/ 1.73 \mathrm{~m}^{2}$ (calculated by MDRD or any other validated formula)

- Alanine aminotransferase/aspartate aminotransferase $\leq 2.5 x$ ULN

NOTE: $\leq 5 x$ ULN is acceptable in case of liver metastases

- Serum lipase $\leq 1.5 x$ ULN

- Platelet count $\geq 75 \times 10^{9} / \mathrm{L}$

- Hemoglobin $\geq 9 \mathrm{~g} / \mathrm{dL}$

- Absolute neutrophil count $\geq 1.5 \times 10^{9} / \mathrm{L}$

- Willingness and ability to comply with scheduled visits and study procedures

- Willingness to participate in accompanying research program

- Collection of current biopsies during screening must be feasible

NOTE: For each patient a FFPE-tumor tissue block must be available for biomarker evaluation. Excisional, incisional or core needle biopsies are appropriate, while fine needle aspirations are insufficient.

-Women of childbearing potential must have a negative pregnancy test result within 7 days before randomization. Women must not be breastfeeding.

- Females who are postmenopausal for at least 1 year before the screening visit OR are surgically sterile OR, if they are of childbearing potential, agree to practice two effective methods of contraception simultaneously, from the time of signing the informed consent until 4 months after the last dose of study drug, or agree to completely abstain from heterosexual intercourse. Males, even if surgically sterilized (i.e., status post vasectomy), who agree to practice effective barrier contraception during the entire study treatment period and until 4 months after the last dose of study drug, OR agree to completely abstain from heterosexual intercourse.

EXCLUSION CRIT - History or presence of pulmonary interstitial disease, drug-related pneumonitis, or radiation pneumonitis at baseline

- Uncontrolled hypertension (hypertension must be adequately treated for control of blood pressure upon study entry)

- Systemic treatment with strong cytochrome P-450 (CYP) 3A inhibitors, strong CYP3A inducers, or moderate CYP3A inducers or treatment with any investigational systemic anticancer agents, chemotherapy or radiation therapy (except stereotactic radiosurgery or stereotactic body radiation therapy) within 14 days of randomization

- Treatment with antineoplastic monoclonal antibodies within 30 days of randomization

- Major surgery within 30 days of randomization. Minor surgical procedures are allowed, such as catheter placement or minimally invasive biopsies.

- Current spinal cord compression (symptomatic or asymptomatic). Patients with leptomeningeal disease without spinal cord compression may participate.

- Significant or uncontrolled cardiovascular disease, specifically including, but not restricted to the following:

- If an acute coronary syndrome has ensued in the past six months, successful reperfusion has to be documented and the patient must be asymptomatic

- New York Heart Association Class III or IV heart failure within six months prior to randomization

- Any history of clinically significant ventricular arrhythmia

- Cerebrovascular accident or transient ischemic attack within six months prior to first dose of study drug

- Malabsorption syndrome or other gastrointestinal illness or condition that could affect oral absorption of the study drug

- Active severe or uncontrolled chronic infection, including but not limited to, the requirement for intravenous antibiotics for longer than two weeks

- History of HIV infection. Testing is not required in the absence of history.

- Chronic hepatitis B (surface antigen-positive) or chronic active hepatitis C infection. Testing is not required in the absence of history.

- Any serious medical condition or psychiatric illness that could, in the investigator's opinion, potentially compromise patient safety or interfere with the completion of treatment according to this protocol

- Known or suspected hypersensitivity to brigatinib or other TKI or their excipients

- Life-threatening illness unrelated to cancer

- Involvement in the planning and/or conduct of the study (applies to both Takeda staff and/or staff of sponsor and study site)

- Patient who might be dependent on the sponsor, site or investigator

- Patient who has been incarcerated or involuntarily institutionalized by court order or by the authorities [§ $40 \mathrm{Abs} .1 \mathrm{~S} .3 \mathrm{Nr} .4$ AMG]

- Patients who are unable to consent because they do not understand the nature, significance and implications of the clinical trial and therefore cannot form a rational intention in the light of the facts [§ 40 Abs. 1 S. 3 Nr. 3a AMG]

- Legal incapacity or limited legal capacity

- Females who are pregnant or breastfeeding

- Patients who have symptomatic CNS metastases (parenchymal or leptomeningeal) at screening or asymptomatic disease 
Table 1 Inclusion and exclusion criteria (Continued)

requiring an increasing dose of corticosteroids to control symptoms within 7 days before randomization.

NOTE: If a patient has worsening neurological symptoms due to CNS metastasis, they must have completed local therapy and be neurologically stable (without requiring an increasing dose of corticosteroids or anticonvulsants) seven days before randomization.

- Rare hereditary galactose intolerance, total lactase deficiency or glucose-galactose malabsorption

molecular tumor profile (e.g. presence of $A L K$ resistance mutations) at the time of disease progression, which will be analyzed in tissue and liquid rebiopsies as part of the study and made available to the investigators using a "fast-track" procedure in order to support therapeutic decisions. Arm A patients failing standard secondgeneration TKIs will also have the option to receive brigatinib as second-line treatment, if so chosen by the treating physician, which will be provided by Takeda.

All TKI will be taken orally at the recommended daily doses, with predefined dose modifications in case of tolerability problems. Safety will be monitored by physical examinations, ECOG performance status, clinical laboratory profiles and continuous assessments of $\mathrm{AE}$ and $\mathrm{QoL}$ will further be evaluated using patient questionnaires (SF-12 and, in case of brain metastases, EORTC QLQBN20). Patients will be allowed to continue study treatment according to the decision of treating physicians for as long as they derive clinical benefit, i.e. radiologic progression per se does not necessitate a treatment switch.

\section{Radiologic assessments}

Baseline tumor evaluation will take place at screening by contrast-enhanced CT of the chest and abdomen. Tumor response will be evaluated according to RECIST
1.1 after two cycles (8 weeks) and every 12 weeks thereafter (Q12W \pm 7 days) in both the first and second line. Moreover, contrast-enhanced CT/MRI of the brain will be performed at baseline testing (MRI is strongly recommended by the study protocol). If intracranial lesions are detected, brain imaging is recommended with every scheduled tumor response assessment thereafter (intervals may be adapted by the investigators depending on the location and size of detected lesions), otherwise brain surveillance will take place with every second radiologic assessment, i.e. 20 weeks after beginning of first or second line, and every 24 weeks thereafter. Baseline disease assessment for the second therapy line must be performed within 30 days prior to treatment start. Assessments will continue until progression, death or initiation of another antineoplastic therapy after the two study treatment lines according to the standard of care (SOC).

\section{Sampling for molecular profiling}

Comprehensive clinical phenotyping and molecular tumor characterization are pivotal to this trial. Tumor biopsies are collected prior to the start of first-line treatment as formalin-fixed paraffin-embedded (FFPE) tissue. Moreover, blood samples will be taken at baseline and
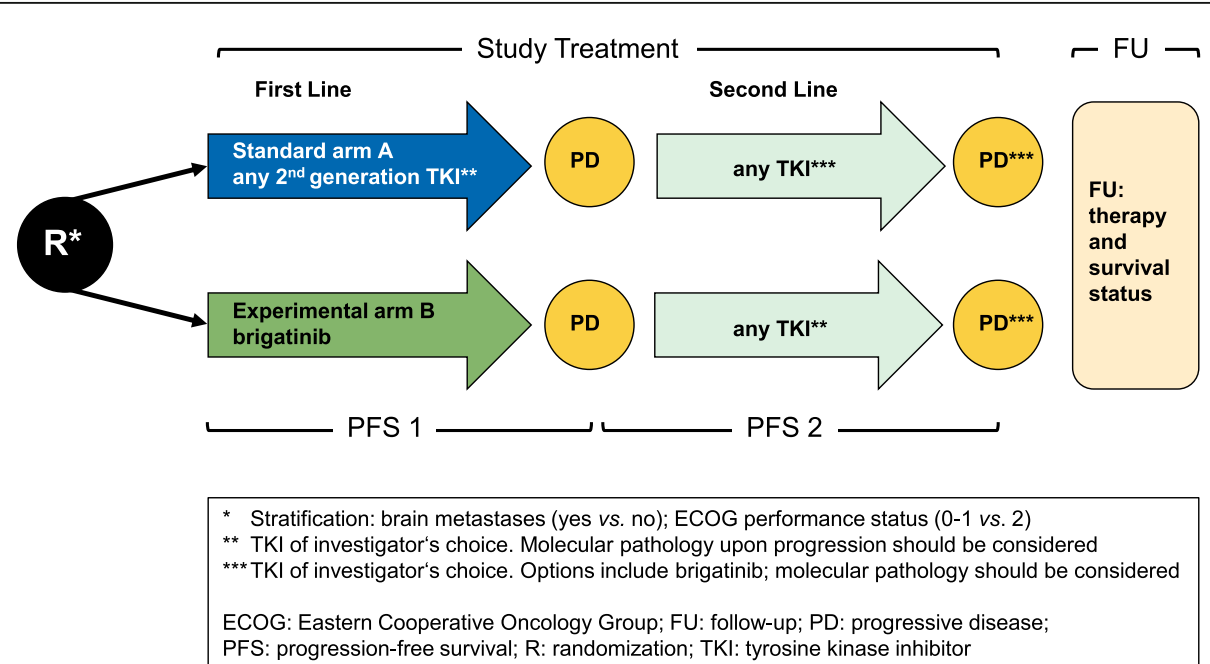

Fig. 1 Study design. Eligible patients are randomized into the two treatment arms stratified by presence of brain metastases and ECOG performance status. First-line treatment in arm B is brigatinib, in arm A it is any other second-generation TKI of the investigator's choice. All subsequent TKI will be chosen by the investigator and should take into account molecular tumor profiles upon progression. After the end of study treatment, patients will be followed-up for toxicity and survival. R: randomization, PD: progressive disease, TKI: tyrosine kinase inhibitor, FU: follow-up 
with every radiologic assessment during the first and second therapy line.

At the time of progression, "fast-track" NGS-based molecular profiling of liquid (ctDNA) and tissue rebiopsies (preferentially on new or growing lesions) will be offered to guide the choice of next-line therapy. In case of isolated intracranial progression, ctDNA-based NGS profiling using cerebrospinal fluid samples will be offered if the patient gives written consent.

An overview of all study procedures is presented in Table 2.

Study management and data quality assurance will be conducted following the standard operational procedures of the Institut für Klinische Krebsforschung IKF GmbH am Krankenhaus Nordwest (Frankfurt, Germany). An eCRF for data collection will be carefully maintained for each participant, also reporting serious and nonserious $\mathrm{AE}$ according to the common criteria for adverse events (CTCAE, version 5.0), and changes of study treatment throughout the trial. Patients will be provided a diary where the study drug intake will be recorded. Missed doses will be captured by the patient diaries and documented in the eCRF.

After the end of the study period, participants will be proactively followed up for survival and/or treatmentrelated AE until loss-to-follow-up or withdrawal of study consent. Patients unwilling to return to the study site will alternatively be offered a follow-up by telephone every three months. The treating physicians will ensure that patients receive appropriate further therapies according to SOC.

\section{Statistical analysis}

Statistical analysis will follow the International Conference on Harmonization (ICH) Guidelines "Structure and Content of Clinical Study Reports" and "Statistical Principles for Clinical Trials".

\section{Sample size and study duration}

The sample size of $n=116$ is primarily determined by considerations of enrollment feasibility. The planned study duration was based on published data from the ALTA-1 L [12] and ALEX [32, 33] phase III trials. In these calculations, the HR for PFS of first-line alectinib vs. crizotinib was set to 0.45 (i.e. the average of 0.47 [32] and 0.43 [33], the HR for PFS of first-line brigatinib vs. crizotinib was assumed identical to that of alectinib vs. crizotinib (based on data of the ALTA- $1 \mathrm{~L}$ trial with $\mathrm{HR}=0.49,95 \%$ confidence intervall (CI) 0.33-0.74 [12]), and the median PFS under first-line crizotinib was considered to be 11 months (i.e. the average of 11.1 [32] and 10.9 months [33]). Consequently, assuming an exponential distribution of PFS, the expected median PFS under first-line alectinib or brigatinib was estimated as 24.4 months (11/0.45), and the proposed duration of the ABP trial was based on an expected total follow-up time of 32 months for the last patient, i.e. an expected first-line PFS of 24.4 months plus an expected second-line PFS of approximately 7 months (based on the median PFS of 5.5-6.9 months [95\% CI 2.9-9.5] under lorlatinib after failure of second-generation ALK inhibitors in the EXP3B/4/5 cohorts of a phase II trial [34]). The recruitment period of 36 months was proposed based on the expected number of newly diagnosed $\mathrm{ALK}^{+}$patients in the centers expected to participate.

To quantify the potential degree of evidence regarding PFS1 that can be gained with a total of 116 patients, we calculated the number of expected events $d$, the expected 95\% CI for the median PFS of alectinib and brigatinib in the first line (assumed to be equal, as explained above), and the expected $95 \%$ CI for the HR of PFS under firstline brigatinib vs. alectinib in the ABP trial, given a constant accrual over a time of 36 months, a follow-up time of 32 months for the last patient, and exponentially distributed PFS times. Under these assumptions, the expected number of PFS events is $d=87$, the expected $95 \%$ CI of the median PFS in the first line is [16.6-34.2 months] (both arms), and the expected 95\% CI of the HR for PFS in the first line [0.66-1.52]. The number of events $d$ was calculated using Schoenfeld's formula [35] and ADDPLAN v6.1 software. The CI for the median PFS was calculated via bootstrapping using 1,000,000 datasets simulated in R v3.3.3 (http://r-project.org) and a fixed random number seed to yield stable and reproducible results, and the $\mathrm{CI}$ for the HR was calculated using the (approximate) formula exp. $( \pm 1.96 \sqrt{ } 4 / \mathrm{d})[36]$.

\section{Methods of statistical analysis}

A Cox proportional hazards model will be used to assess the primary endpoint PFS1. As covariates, the model includes the factor "treatment group" and is adjusted for the presence of brain metastases (yes vs. no) and ECOG $(0-1$ vs. 2) at baseline. The treatment groups will be compared at a two-sided $\alpha$ of 0.05 , and a $95 \% \mathrm{CI}$ for the HR determined. Furthermore, Kaplan-Meier curves will be provided. Primary analysis will be based on all randomized patients (intention-to-treat population), while sensitivity analyses will be conducted for the perprotocol set (patients without major protocol violations) and for predefined subgroups of secondary and exploratory endpoints.

Analyses of secondary endpoints will be descriptive and will include the calculation of appropriate summary measures of the empirical distributions. AE and SAE will be summarized by relative and absolute frequency and severity grade based on CTCAE V5.0. Summary tables will provide the number and percentage of patients with $\mathrm{AE}$ and the $95 \% \mathrm{CI}$ for the event rates. 
Table 2 Schedule of Study Assessments and Procedures

\begin{tabular}{|c|c|c|c|c|c|c|c|c|}
\hline \multirow[t]{2}{*}{ Assessment } & \multicolumn{3}{|c|}{ Screening period } & \multicolumn{4}{|c|}{$\begin{array}{l}\text { Study treatment period ( } 1 \text { st -and } 2 \text { nd- } \\
\text { line treatment, respectively) * }\end{array}$} & \multirow[t]{2}{*}{$\begin{array}{l}\text { Follow- } \\
\text { Up }\end{array}$} \\
\hline & \multicolumn{3}{|c|}{$\begin{array}{l}\text { Screening } \\
\text { (up to } 28 \text { days prior to D1) }\end{array}$} & $\begin{array}{l}\text { Cycle } 1 \\
\text { a }\end{array}$ & $\begin{array}{l}\text { Cycle } \\
2^{\mathrm{a}}\end{array}$ & $\begin{array}{l}\text { Cycle } 3 \text { to Cycle } \\
\mathrm{n}^{\mathrm{a}}\end{array}$ & EOT & \\
\hline Day / Frequency & $\begin{array}{l}\text { D-28 to } \\
\text { D0 }\end{array}$ & $\begin{array}{l}\text { D-14 to } \\
\text { D0 }\end{array}$ & $\begin{array}{l}\text { D-7 to } \\
\text { D0 }\end{array}$ & D1 D8 & D1 & $\begin{array}{l}\text { D1 or D15 } \\
\text { alternating } \\
(\mathrm{Q} 6 \mathrm{~W} \pm 3 \mathrm{~d})\end{array}$ & & $\begin{array}{l}\text { Q12W } \\
\pm 21 \\
\text { days }\end{array}$ \\
\hline
\end{tabular}

Informed consent, eligibility criteria, demographics, medical / $\mathrm{X}$ surgical history ${ }^{c}$

\section{Examinations}

$P E^{e}$

Height

Vital signs ${ }^{\circ}$, Weight

ECG

ECOG Performance Status

\section{Laboratory}

\author{
Hematology $^{p}$ \\ Chemistry $^{p}$ \\ Coagulation $^{\mathrm{p}}$ \\ Pregnancy test ${ }^{i}$ \\ Cerebrospinal fluid (local lab) \\ (optional)
}

\section{Study treatment}

Arm A - 1st line:

Any 2nd-generation TKI

Arm B - 1st line:

Brigatinib

Arm A - 2nd line:

Any TKI

Arm B - 2nd line:

Any TKI

Diary review with patient ${ }^{k}$

\section{Continuous assessments}

Disease assessment ${ }^{a}$

QoL $^{j}$

$\mathrm{AE} /$ toxicities

Concomitant medications

\section{Biomarker sampling}

Biopsy (FFPE tumor tissue) ${ }^{\mathbf{d}}$

Blood sample collection $^{n}$

Cerebrospinal fluid $x$

$x$ $\underset{f, g}{X} \underset{g, m}{X} \quad X \quad X$

$\begin{array}{llllll} & x^{f} & x & x & x\end{array}$

Whenever clinically indicated $\quad x$

$x^{f} \quad x \quad x \quad x$

$\begin{array}{llll}x^{f} & x & x & x\end{array}$

$\begin{array}{lllll}x^{f} & x^{h} & x & x & x\end{array}$

$x$

X $\quad X^{f}$ Every 12 weeks (Q12W \pm 7 days) $\quad X$

Recommended for "brain-only" progression at time of PD

Dose and frequency according to current SmPC

Once daily oral dose (QD p.o.)

[90 mg D1 to D7, $180 \mathrm{mg}$ from D8

g,m]

Dose and frequency according to current SmPC

Dose and frequency according to current SmPC

$$
\begin{array}{lll}
x^{k} & x \quad x & x
\end{array}
$$

Throughout study ${ }^{a}$

$X \quad$ Together with imaging of chest and $\quad X$ abdomen

Throughout study at every treatment day

Throughout study

After failure of first-line TKI ${ }^{d}$ After failure of second-line TKI ${ }^{d}$

X Throughout study ${ }^{n}$

Recommended for "brain-only" progression (at time of PD) 
Table 2 Schedule of Study Assessments and Procedures (Continued)

\begin{tabular}{|c|c|c|c|c|c|c|c|c|}
\hline \multirow{3}{*}{$\begin{array}{l}\text { Assessment } \\
\text { Day / Frequency }\end{array}$} & \multirow{2}{*}{\multicolumn{3}{|c|}{$\begin{array}{l}\text { Screening period } \\
\text { Screening } \\
\text { (up to } 28 \text { days prior to } D 1 \text { ) }\end{array}$}} & \multicolumn{4}{|c|}{$\begin{array}{l}\text { Study treatment period ( } 1 \text { st -and } 2 \text { nd- } \\
\text { line treatment, respectively) }{ }^{*}\end{array}$} & \multirow{3}{*}{$\begin{array}{l}\text { Follow- } \\
\text { Up } \\
\\
\text { Q12W } \\
\pm 21 \\
\text { days }\end{array}$} \\
\hline & & & & $\begin{array}{l}\text { Cycle } 1 \\
\text { a }\end{array}$ & \multirow{2}{*}{$\begin{array}{l}\text { Cycle } \\
2^{a} \\
\text { D1 }\end{array}$} & \multirow{2}{*}{$\begin{array}{l}\text { Cycle } 3 \text { to Cycle } \\
\mathrm{n}^{\text {a }} \\
\text { D1 or D15 } \\
\text { alternating } \\
(\mathrm{Q} 6 \mathrm{~W} \pm 3 \mathrm{~d})\end{array}$} & \multirow[t]{2}{*}{ EOT } & \\
\hline & $\begin{array}{l}\text { D-28 to } \\
\text { D0 }\end{array}$ & $\begin{array}{l}\text { D-14 to } \\
\text { Do }\end{array}$ & $\begin{array}{l}\text { D-7 to } \\
\text { D0 }\end{array}$ & D1 D8 & & & & \\
\hline \multicolumn{9}{|l|}{ Follow-up assessments } \\
\hline Survival $^{\prime}$ & & & & & & & & $x^{1}$ \\
\hline Subsequent anticancer therapy & & & & & & & & $x$ \\
\hline
\end{tabular}

* Second-line treatment is started after a washout period of 3 days. Choice of next-line treatment should take into account molecular results of rebiopsies. Disease assessment, including baseline imaging for the second line, must be performed within 30 days before therapy start

${ }^{a} A$ cycle is defined as 28 days; acceptable window for regular visits: \pm 3 days, starting from cycle 2

${ }^{\mathrm{b}}$ EOT visits 30 days $( \pm 7 \mathrm{~d})$ after the last dose of first- and second-line treatment (30 days safety follow-up)

${ }^{c}$ Medical history with details on any prior NSCLC therapy, including start and end date(s), best response(s). NOTE: Assay and sample type of prior ALK tests must be documented

dExploratory biomarker studies: Central NGS-based multiplex analysis is mandatory for baseline FFPE-tissue biopsies and recommended for lesions growing under therapy

${ }^{\text {e}}$ Complete physical examination (PE) at screening and EOT; symptom-directed PE at any other time point (at investigator's discretion)

${ }^{f} \mathrm{PE}$, ECOG performance status assessments, hematology, chemistry, and pregnancy tests are required at C1D1 (unless performed within 7 days before and no changes anticipated)

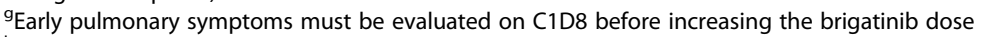

${ }^{\mathrm{h}}$ Aspartate aminotransferase / alanine aminotransaminase and total bilirubin only

iPregnancy tests (urine or serum beta-human chorionic gonadotropin only, obligatory for women of childbearing potential) must be confirmed negative within 7 days before TKI start (i.e., 7 days prior to C1D1). To be repeated once every 12 weeks thereafter (every 3 cycles; Q12W \pm 7 days) and at EOT visit (additional testing if indicated)

${ }^{\mathrm{j}} \mathrm{QoL}$ assessments at baseline, all tumor assessment visits (refer to footnote q), and at EOT visit (SF-12, and, in case of brain involvement, also EORTC QLQ-BN20) kPatients must document TKI intake. On C1D8, on D1 of each cycle and at the EOT visit, site staff reviews diary and crosschecks tablets or tablet bottles in the patient's possession

Survival follow-up shall be scheduled $Q 12 W \pm 21$ days until the end of the study

mpatients tolerating the brigatinib starting dose (90 mg, C1D1-D7) should increase the dose to $180 \mathrm{mg}$ QD from C1D8

${ }^{n}$ Blood samples at baseline (i.e., up to 7 days before TKI start) and with every CT/MRI assessment during active first- and second-line therapy (i.e. two cycles [8 weeks] after TKI start and every 12 weeks thereafter [Q12W \pm 7 days] (at every CT/MRI assessment visit). Samples: 2 x $7.5 \mathrm{ml}$ EDTA blood and 1x 7.5 ml serum. In addition, $2 \times 2.5 \mathrm{ml}$ PAXgene blood for RNA isolation at four time points: i.e., (1) up to 7 days before start of first-line TKI, (2) following five cycles of first-line TKI [at the second CT/MRI assessment], (3) up to 7 days before start of second-line TKI, (4) after five cycles of second-line TKI [with CT/MRI assessment]

${ }^{\circ}$ Vital signs: body temperature, pulse, blood pressure, oxygen saturation. Special attention must be paid to blood pressure monitoring at screening and during treatment. Patients with controlled hypertension at inclusion must receive appropriate antihypertensive treatment during study participation (antihypertensive medication at the investigator's discretion, to be documented in the subject's Concomitant Medication eCRF)

${ }^{P}$ Laboratory assessments may be performed up to one day before the visit (entire study) in order to have results available on the visit day. Hematology panel: Complete Blood Cell (CBC) count with differential. Chemistry panel: albumin; alkaline phosphatase; alanine aminotransferase; aspartate aminotransferase; serum lipase, serum amylase; lactate dehydrogenase; creatine kinase; creatinine; calcium; glucose; phosphate; potassium; sodium; magnesium; total bilirubin; blood urea nitrogen or blood urea; C-reactive protein; gamma-glutamyltransferase. Coagulation: INR or PTT only at baseline

${ }^{\mathrm{q}}$ Contrast-enhanced CT/MRI (chest/abdomen): all patients (unless contraindicated). Tumor response is evaluated according to RECIST 1.1 (investigator assessment). Baseline tumor evaluation at screening; response assessment according to SOC, i.e. after two cycles ( 8 weeks) and every 12 weeks thereafter (Q12W \pm 7 days) during active treatment in the first and second line. Second-line: baseline disease assessment should be performed within 30 days before TKI start. Intracranial response evaluation according to RECIST 1.1 (all patients): contrast-enhanced brain MRI/CT at screening (MRI strongly recommended). In case of brain metastasis, imaging is recommended with every scheduled assessment (intervals may be adapted depending on location and size of lesions), otherwise brain surveillance should take place according to SOC (with every second radiologic assessment). Assessments will continue until progression, death or initiation of another antineoplastic therapy after the two study treatment lines according to SOC

A solely descriptive interim evaluation to assess the primary (PFS of first-line treatment) and secondary (iORR, iDOR, iTTP, TNT of first line treatment, PFS of second-line treatment, TNT of second line treatment, OS, QoL, frequency of SAE) endpoints will be performed when 25 patients in each arm have a follow-up of at least 12 months.

All analyses will be carried out using SAS (SAS Institute, Cary, NC) version 9.4 or higher.

\section{Trial status}

As of June 21, 2021, 25 study centers have been initiated. The first patient was enrolled on June 18, 2020.

\section{Discussion}

The ABP trial has been designed to provide insights and generate hypotheses that will facilitate optimization of management for patients with $\mathrm{ALK}^{+}$NSCLC. Besides supporting therapeutic decisions for enrolled patients by providing "fast-track" molecular results to the treating physicians, the ABP trial also aims to deepen understanding of the underlying biology and facilitate development of a framework for individualized management of $\mathrm{ALK}^{+}$NSCLC according to molecular properties. One special focus is the relationship of resistance mechanisms acquired under treatment with the therapeutic compound used and baseline tumor characteristics. Patients with low molecular risk and the perspective of a 
"chronic disease" will be distinguished from "high-risk" cases, and molecular features of the latter will be utilized to elaborate improved non-invasive methods of disease monitoring and preclinical modeling in order to advance therapeutic strategies.

\section{Abbreviations}

AE: Adverse event; ALK: Anaplastic lymphoma kinase; Cl: Confidence interval; CNS: Central nervous system; CT: Computed tomography; CTCAE: Common Terminology Criteria for Adverse Event; ctDNA: Circulating tumor DNA; DOR: Duration of response; ECOG: Eastern Cooperative Oncology Group: eCRF: Electronic case report form; EGFR: Epidermal growth factor receptor; EML4: Echinoderm microtubule-associated protein-like 4; EOT: End of treatment; FFPE: Formalin-fixed, paraffin embedded; HR: Hazard ratio; ICH: International Council for Harmonisation; iDoR: Intracranial duration of response; IORR: Intracranial objective response rate; KRAS: Kirsten rat sarcoma viral oncogene homolog; MRI: Magnetic resonance imaging; NGS: Nextgeneration sequencing; NSCLC: Non-small cell lung cancer; OS: Overall survival; PFS: Progression-free survival; PE: Physical examination; QoL: Quality of life; RECIST 1.1: Response Evaluation Criteria in Solid Tumors, version 1.1; SAE: Serious adverse event; SmPC: Summary of Product Characteristics; SOC: Standard of care; TKI: Tyrosine kinase inhibitor; TNT: Time to next treatment; TP53: Tumor Protein 53; iTTP: Time to intracranial progression

\section{Acknowledgements}

This trial is conducted in cooperation with the Thoracic Oncology Working Group of the Arbeitsgemeinschaft Internistische Onkologie (AIO) within the German Cancer Society. We acknowledge financial support for this publication by the Baden-Württemberg Ministry of Science, Research and the Arts and by the Ruprecht-Karls-University Heidelberg.

\section{Authors' contributions}

MT, PC, FB, IC, LB developed the study idea, wrote the protocol and coordinated funding, regulatory permission and approval processes. MT is lead investigator of this study; PC is deputy lead investigator. JK calculated (bio-) statistical models for sample size, study hypothesis, and endpoint determinations. MS developed blood-based biomarker evaluation strategies and will perform all respective analyses. AS will conduct tissue- and ctDNAbased NGS assessments. The Institut für Klinische Krebsforschung IKF GmbH am Krankenhaus Nordwest (RE, DM) is legal sponsor of the study, gave advice on study design and is responsible for study management and logistics. All authors have read and approved the current version of the manuscript.

\section{Authors' information}

not provided.

\section{Funding}

The Institut für Klinische Krebsforschung IKF GmbH am Krankenhaus Nordwest (Frankfurt, Germany), an independent research organization, acts as the legal sponsor of the trial. Financial support and brigatinib are provided by Takeda. Open Access funding enabled and organized by Projekt DEAL.

\section{Availability of data and materials}

Data generated by this study will be made available by the corresponding author on reasonable request.

\section{Declarations}

\section{Ethics approval and consent to participate}

This clinical trial has been approved by the Ethics Committee of the Medical Faculty of the University of Heidelberg (AFmu-701/2019, February 21, 2020). Before participation in the trial, written informed consent is obtained from each subject according to the ICH guidelines on Good Clinical Practice. Additionally, the Bundesinstitut für Arzneimittel und Medizinprodukte- BfArM (Federal Institute for Drugs and Medical Devices, the medical regulatory body of the German Federal Ministry of Health) has approved the study (January 03, 2020).

\section{Consent for publication}

Not applicable, as no individual patient data are contained in this manuscript.

\section{Competing interests}

The ABP trial receives funding from the pharmaceutical company Takeda. However, Takeda has not been involved in study design, data collection, management, data analysis and interpretation, or in the decision to submit this protocol for publication. PC reports research funding from AstraZeneca, Novartis, Roche, Takeda, and advisory board/lecture fees from AstraZeneca, Boehringer Ingelheim, Chugai, Novartis, Pfizer, Roche, Takeda. FB reports research funding from BMS and travel grants from BMS and MSD. AS receives advisory board honoraria from BMS, AstraZeneca, ThermoFisher, Novartis, speaker's honoraria from BMS, Illumina, AstraZeneca, Novartis, ThermoFisher, MSD, Roche, and research funding from Chugai. MT reports advisory board honoraria from Novartis, Lilly, BMS, MSD, Roche, Celgene, Takeda, AbbVie, Boehringer, speaker's honoraria from Lilly, MSD, Takeda, research funding from AstraZeneca, BMS, Celgene, Novartis, Roche and travel grants from BMS, MSD, Novartis, Boehringer. All other authors declare no conflicts of interest.

\section{Author details}

'Department of Thoracic Oncology, Thoraxklinik at University Hospital of Heidelberg, Röntgenstraße 1, 69126 Heidelberg, Germany. ${ }^{2}$ Translational Lung Research Center Heidelberg TLRCH, Member of the German Center for Lung Research DZL, Im Neuenheimer Feld 156, 69120 Heidelberg, Germany. ${ }^{3}$ University Hospital of Heidelberg, Institute of Medical Biometry and Informatics, Im Neuenheimer Feld 130.3, 69120 Heidelberg, Germany. ${ }^{4}$ Translational Research Unit (STF), Thoraxklinik at University Hospital of Heidelberg, Röntgenstraße 1, 69126 Heidelberg, Germany. ${ }^{5}$ University of Heidelberg, Institute of Pathology, Im Neuenheimer Feld 224, 69120 Heidelberg, Germany. ${ }^{6}$ Institut für Klinische Krebsforschung IKF GmbH am Krankenhaus Nordwest, Steinbacher Hohl 2-26, 60488 Frankfurt am Main, Germany.

Received: 25 November 2020 Accepted: 8 June 2021

Published online: 28 June 2021

\section{References}

1. Jemal A, Bray F, Center MM, Ferlay J, Ward E, Forman D. Global cancer statistics. CA Cancer J Clin. 2011;61(2):69-90. https://doi.org/10.3322/caac.2 0107.

2. Planchard D, Popat S, Kerr K, Novello S, Smit EF, Faivre-Finn C, et al. Metastatic non-small cell lung cancer: ESMO Clinical Practice Guidelines for diagnosis, treatment and follow-up. Ann Oncol. 2018;29(Suppl 4):iv192-237.

3. Siegel RL, Miller KD, Jemal A. Cancer statistics, 2020. CA Cancer J Clin. 2020; 70(1):7-30. https://doi.org/10.3322/caac.21590.

4. Devarakonda S, Morgensztern D, Govindan R. Genomic alterations in lung adenocarcinoma. Lancet Oncol. 2015;16(7):e342-51. https://doi.org/10.1016/ S1470-2045(15)00077-7.

5. Shaw AT, Engelman JA. ALK in lung cancer: past, present, and future. J Clin Oncol. 2013;31(8):1105-11. https://doi.org/10.1200/JCO.2012.44.5353.

6. Christopoulos P, Budczies J, Kirchner M, Dietz S, Sultmann H, Thomas M, et al. Defining molecular risk in ALK(+) NSCLC. Oncotarget. 2019;10(33): 3093-103. https://doi.org/10.18632/oncotarget.26886.

7. Spigel DR, Schrock AB, Fabrizio D, Frampton GM, Sun J, He J, et al. Total mutation burden $(T M B)$ in lung cancer $(\mathrm{LC})$ and relationship with response to PD-1/PD-L1 targeted therapies. J Clin Oncol. 2016;34(15_suppl):9017-7.

8. Mok T, Camidge DR, Gadgeel SM, Rosell R, Dziadziuszko R, Kim DW, et al. Updated overall survival and final progression-free survival data for patients with treatment-naive advanced ALK-positive non-small-cell lung cancer in the ALEX study. Ann Oncol. 2020;31(8):1056-64. https://doi.org/10.1016/j.a nnonc.2020.04.478.

9. Solomon BJ, Kim D-W, Wu Y-L, Nakagawa K, Mekhail T, Felip E, et al. Final overall survival analysis from a study comparing first-line Crizotinib versus chemotherapy in ALK-mutation-positive non-small-cell lung Cancer. J Clin Oncol. 2018;36(22):2251-8. https://doi.org/10.1200/JCO.2017.77.4794.

10. Ramalingam SS, Vansteenkiste J, Planchard D, Cho BC, Gray JE, Ohe Y, et al. Overall survival with Osimertinib in untreated, EGFR-mutated advanced NSCLC. N Engl J Med. 2020;382(1):41-50. https://doi.org/10.1056/NEJMoa1 913662. 
11. Camidge DR, Kim HR, Ahn MJ, Yang JCH, Han JY, Hochmair MJ, Lee KH, Delmonte A, García Campelo MR, Kim DW, Griesinger F, Felip E, Califano R, Spira A, Gettinger SN, Tiseo M, Lin HM, Gupta N, Hanley MJ, Ni Q, Zhang P, Popat S. Brigatinib Versus Crizotinib in Advanced ALK Inhibitor-Naive ALKPositive Non-Small Cell Lung Cancer: Second Interim Analysis of the Phase III ALTA-1L Trial. J Clin Oncol. 2020;38(31):3592-3603. https://doi.org/10.1200/ JCO.20.00505. Epub 2020 Aug 11.

12. Camidge DR, Kim HR, Ahn MJ, Yang JC, Han JY, Lee JS, et al. Brigatinib versus Crizotinib in ALK-positive non-small-cell lung Cancer. N Engl J Med. 2018;379(21):2027-39. https://doi.org/10.1056/NEJMoa1810171.

13. Planchard D, Popat S, Kerr K, Novello S, Smit EF, Faivre-Finn C, et al. Metastatic non-small cell lung cancer: ESMO clinical practice guidelines for diagnosis, treatment and follow-up. Ann Oncol. 2019;30(5):863-70. https:// doi.org/10.1093/annonc/mdy474.

14. ESMO Clinical Practice Living Guidelines - Metastatic Non-Small-Cell Lung Cancer. https://www.esmo.org/guidelines/lung-and-chest-tumours/clinicalpractice-living-guidelines-metastatic-non-small-cell-lung-cancer. Accessed Nov 2020.

15. Solomon B, Bauer TM, De Marinis F, Felip E, Goto Y, Liu G, et al. LBA2 Lorlatinib vs crizotinib in the first-line treatment of patients (pts) with advanced ALK-positive non-small cell lung cancer (NSCLC): results of the phase III CROWN study. Ann Oncol. 2020;31:S1180-1. https://doi.org/10.101 6/j.annonc.2020.08.2282.

16. Woo CG, Seo S, Kim SW, Jang SJ, Park KS, Song JY, et al. Differential protein stability and clinical responses of EML4-ALK fusion variants to various ALK inhibitors in advanced ALK-rearranged non-small cell lung cancer. Ann Oncol. 2017;28(4):791-7. https://doi.org/10.1093/annonc/mdw693.

17. O'Regan L, Barone G, Adib R, Woo CG, Jeong HJ, Richardson EL, Richards MW, Muller PAJ, Collis SJ, Fennell DA, Choi J, Bayliss R, Fry AM. EML4- ALK V3 oncogenic fusion proteins promote microtubule stabilization and accelerated migration through NEK9 and NEK7. J Cell Sci. 2020;133(9): jes241505. https://doi.org/10.1242/jcs.241505.

18. Christopoulos P, Endris V, Bozorgmehr F, Elsayed M, Kirchner M, Ristau J, et al. EML4-ALK fusion variant V3 is a high-risk feature conferring accelerated metastatic spread, early treatment failure and worse overall survival in ALK+ non-small cell lung cancer. Int J Cancer. 2018;142(12):258998. https://doi.org/10.1002/ijc.31275

19. Christopoulos P, Kirchner M, Endris V, Stenzinger A, Thomas M. EML4-ALK V3, treatment resistance, and survival: refining the diagnosis of ALK + NSCLC. J Thorac Dis. 2018;10(S16):S1989-91. https://doi.org/10.21037/jtd.2 018.05.61.

20. Bayliss R, Choi J, Fennell DA, Fry AM, Richards MW. Molecular mechanisms that underpin EML4-ALK driven cancers and their response to targeted drugs. Cell Mol Life Sci. 2016;73(6):1209-24. https://doi.org/10.1007/s00018015-2117-6.

21. Kron A, Alidousty C, Scheffler M, Merkelbach-Bruse S, Seidel D, Riedel R, et al. Impact of TP53 mutation status on systemic treatment outcome in ALK-rearranged non-small-cell lung cancer. Ann Oncol. 2018;29(10):2068-75. https://doi.org/10.1093/annonc/mdy333.

22. Christopoulos $P$, Kirchner $M$, Bozorgmehr $F$, Endris $V$, Elsayed M, Budczies J, et al. Identification of a highly lethal V3(+) TP53(+) subset in ALK(+) lung adenocarcinoma. Int J Cancer. 2019;144(1):190-9. https://doi.org/10.1002/ ijc.31893.

23. Christopoulos $P$, Dietz $S$, Kirchner M, Volckmar AL, Endris V, Neumann O, Ogrodnik S, Heussel CP, Herth FJ, Eichhorn M, Meister M, Budczies J, Allgäuer M, Leichsenring J, Zemojtel T, Bischoff $H$, Schirmacher P, Thomas M, Sültmann H, Stenzinger A. Detection of TP53 Mutations in Tissue or Liquid Rebiopsies at Progression Identifies ALK+ Lung Cancer Patients with Poor Survival. Cancers (Basel). 2019;11(1):124. https://doi.org/10.3390/ca ncers11010124.

24. Camidge DR, Niu H, Kim HR, Yang JC-H, Ahn M-J, Li JY-C, et al. Correlation of baseline molecular and clinical variables with ALK inhibitor efficacy in ALTA-1L. J Clin Oncol. 2020;38(15_suppl):9517-7.

25. McCoach CE, Blakely CM, Banks KC, Levy B, Chue BM, Raymond VM, et al. Clinical utility of cell-free DNA for the detection of ALK fusions and genomic mechanisms of ALK inhibitor resistance in non-small cell lung Cancer. Clin Cancer Res. 2018;24(12):2758-70. https://doi.org/10.1158/10780432.CCR-17-2588.

26. Gainor JF, Dardaei L, Yoda S, Friboulet L, Leshchiner I, Katayama R, et al. Molecular mechanisms of resistance to first- and second-generation ALK inhibitors in ALK-rearranged lung Cancer. Cancer Discov. 2016;6(10):111833. https://doi.org/10.1158/2159-8290.CD-16-0596.

27. Dietz S, Christopoulos P, Yuan Z, Angeles AK, Gu L, Volckmar AL, Ogrodnik SLJ, Janke F, Fratte CD, Zemojtel T, Schneider MA, Kazdal D, Endris V, Meister M, Muley T, Cecchin E, Reck M, Schlesner M, Thomas M, Stenzinger A, Sültmann H. Longitudinal therapy monitoring of ALK-positive lung cancer by combined copy number and targeted mutation profiling of cell-free DNA. EBioMedicine. 2020;62:103103.

28. Eisenhauer EA, Therasse P, Bogaerts J, Schwartz LH, Sargent D, Ford R, et al. New response evaluation criteria in solid tumours: revised RECIST guideline (version 1.1). Eur J Cancer. 2009;45(2):228-47. https://doi.org/10.1016/j.ejca.2 008.10.026.

29. Buttner R, Wolf J, Kron A, Nationales Netzwerk Genomische M. The national Network Genomic Medicine (nNGM) : Model for innovative diagnostics and therapy of lung cancer within a public healthcare system. Pathologe. 2019; 40(3):276-80.

30. Volckmar AL, Leichsenring J, Kirchner M, Christopoulos P, Neumann O, Budczies J, et al. Combined targeted DNA and RNA sequencing of advanced NSCLC in routine molecular diagnostics: analysis of the first 3,000 Heidelberg cases. Int J Cancer. 2019;145(3):649-61. https://doi.org/10.1002/ ijc.32133.

31. Mino-Kenudson M, Chirieac LR, Law K, Hornick JL, Lindeman N, Mark EJ, et al. A novel, highly sensitive antibody allows for the routine detection of ALK-rearranged lung adenocarcinomas by standard immunohistochemistry. Clin Cancer Res. 2010;16(5):1561-71. https://doi.org/10.1158/1078-0432.CCR09-2845.

32. Peters S, Camidge DR, Shaw AT, Gadgeel S, Ahn JS, Kim DW, et al. Alectinib versus Crizotinib in untreated ALK-positive non-small-cell lung Cancer. N Engl J Med. 2017;377(9):829-38. https://doi.org/10.1056/NEJMoa1704795.

33. Camidge DR, Dziadziuszko R, Peters S, Mok T, Noe J, Nowicka M, et al. Updated efficacy and safety data and impact of the EML4-ALK fusion variant on the efficacy of Alectinib in untreated ALK-positive advanced nonsmall cell lung Cancer in the global phase III ALEX study. J Thorac Oncol. 2019;14(7):1233-43. https://doi.org/10.1016/j.jtho.2019.03.007.

34. Solomon B, Shaw A, Ou S. OA 05.06 Phase 2 Study of Lorlatinib in Patients with Advanced ALK+/ROS1+ Non-Small-Cell Lung Cancer. J Thorac Oncol. 2017;12(11):1756.

35. Schoenfeld D. The asymptotic properties of nonparametric tests for comparing survival distributions. Biometrika. 1981;68(1):316-9. https://doi. org/10.1093/biomet/68.1.316

36. Wassmer G. Planning and analyzing adaptive group sequential survival trials. Biom J. 2006;48(4):714-29. https://doi.org/10.1002/bimj.200510190.

\section{Publisher's Note}

Springer Nature remains neutral with regard to jurisdictional claims in published maps and institutional affiliations.

\section{Ready to submit your research? Choose BMC and benefit from:}

- fast, convenient online submission

- thorough peer review by experienced researchers in your field

- rapid publication on acceptance

- support for research data, including large and complex data types

- gold Open Access which fosters wider collaboration and increased citations

- maximum visibility for your research: over $100 \mathrm{M}$ website views per year

At BMC, research is always in progress.

Learn more biomedcentral.com/submissions 